which are form-invariant under the full orthogonal group $\dagger$. Since $P^{\prime}$ is linear in the vectors, we can omit those elements $u_{\alpha}^{(r)} u_{\alpha}^{(s)}$ for which $r=s$. We thus have the result that the quantities

$$
u_{3}^{(r)}, u_{\alpha}^{(r)} u_{\alpha}^{(s)} \quad(r, s=1,2, \cdots, n ; r \neq s)
$$

form a polynomial basis for polynomials in the components of $n$ vectors $u_{i}^{(r)}$ which are linear in each of the vectors and form-invariant under the transformations associated with transverse isotropy about the $x_{3}$ axis.

As in the previous sections, we immediately see that a basic set of anisotropic tensors for transverse isotropy about the $x_{3}$ axis is defined by $\partial u_{3}^{(r)} / \partial u_{i}^{(r)}\left(=\alpha_{i}\right.$, say) and $\partial\left(u_{\alpha}^{(r)} u_{\alpha}^{(e)} / \partial u_{i}^{(r)} \partial u_{i}^{(o)}\left(=\beta_{i j}\right)(r \neq s ; \alpha=1,2 ; i, j=1,2,3)\right.$. We obtain immediately

$$
\left(\alpha_{i}\right)=\left(\delta_{3 i}\right)=(0,0,1)
$$

and

$$
\left(\beta_{i j}\right)=\left(\delta_{1 i} \delta_{1 i}+\delta_{2 i} \delta_{2 i}\right)=\left[\begin{array}{lll}
1, & 0, & 0 \\
0, & 1, & 0 \\
0, & 0, & 0
\end{array}\right) .
$$

\title{
ON A PRINCIPLE OF RECIPROCITY BETWEEN HIGH- AND LOW-FREQUENCY PROBLEMS CONCERNING LINEAR DIFFERENTIAL EQUATIONS OF SECOND ORDER*
}

\section{BY AUREL WINTNER (The Johns Hopkins University)}

1. The following considerations, in which nomenclature and point of view will be the same as in two earlier notes ${ }^{1}$, deal with the oscillatory case of the differential equation

$$
x^{\prime \prime}+f(t) \dot{x}=0,
$$

in which $f(t)$ is a real-valued, continuous function for large positive $t$, and $x(t)$ is any real-valued solution distinct from the trivial solution $x(t) \equiv 0$.

If (1) is oscillatory when $f(t)=f_{1}(t)$, and if $f_{1}(t) \leqq f_{2}(t)$, then (1) is oscillatory when $f(t)=f_{2}(t)$. This follows from Sturm's comparison theorem, which implies also the following fact: If (1) is oscillatory and if $d_{n}=d_{n}(f)=d_{n}(f ; x)$ denotes the distance $t_{n+1}-t_{n}$, where $t_{n}=t_{n}(f ; x)$ is the $n$th zero of a solution $x=x(t)=x_{f}(t)$ of $(1)$, then lim sup $d_{n}\left(f_{1}\right) / d_{n}\left(f_{2}\right)<1$, where $n \rightarrow \infty$, holds whenever $f_{2}(t)-f_{1}(t)$ exceeds a positive lower bound as $t \rightarrow \infty$.

By choosing $f_{1}(t)=\omega^{2}$, where $\omega$ is a positive constant, and letting $\omega \rightarrow \infty$ or $\omega \rightarrow 0$, it follows that $d_{n}(f) \rightarrow 0$ as $n \rightarrow \infty$ if

$$
f(t) \rightarrow \infty \quad \text { as } t \rightarrow \infty
$$

†See, for example, H. Weyl, loc. cit. p. 52 et. seq.

*Received July 5, 1956.

1A. Wintner, Quart. Appl. Math. 7, 115-117 (1949) and 13, 192-195 (1955). These two papers will be referred to as [1] and [2] respectively. 
and that $d_{n}(f) \rightarrow \infty$ as $n \rightarrow \infty$ if

$$
f(t) \rightarrow 0 \text { as } t \rightarrow \infty
$$

(provided that (1) is oscillatory; this additional proviso is necessary, even if

$$
f(t)>0,
$$

in the case (3), but is automatically satisfied in the case (2), or even if just $f(t) \geqq \omega^{2}$ holds for some positive constant.) For this reason, (1) will be called of high frequency in the (oscillatory subcase of the) case (3).

2. If $f(t)=\lambda / t^{2}$ (for $\left.1 \leqq t<\infty\right)$, where $\lambda$ is a positive constant, then (4) and (3) are satisfied (and

$$
\int^{\infty} f(t) d t=\infty
$$

fails to hold). But it depends on the numerical value of $\lambda$ (hence, not merely on the order of magnitude of $f(t)>0$ for large $t$ ) whether (1) is or is not oscillatory in this case. In fact, the remark of $\mathrm{A}$. Kneser, quoted in ${ }^{2}$ [2], shows that the case $f(t)=\lambda / t^{2}$ of (1) is oscillatory only if $\lambda>1 / 4$, rather than just $\lambda>0$, is assumed.

Thus (3) and the negation of (5) together are compatible with the oscillatory as well as with the non-oscillatory character of (1) in the case (4). On the other hand, (1) must be oscillatory if (4) and (5) hold; in fact, more than this was proved in [1].

In what follows, both (4) and (5) will be assumed. This will imply, in particular, that (1) is oscillatory even if (3) holds.

3. There is an extensive literature ${ }^{3}$ dealing with the asymptotic behavior (as $t \rightarrow \infty$ ) of the solutions $x(t)$ of (1) in the case (2) of high frequency. Let any such theorem be referred to as of type $(H)$, and let $(L)$ refer to theorems dealing with the asymptotic behavior of the solutions $x(t)$ of (1) in the case (3) of low frequency.

An inspection of the literature reveals a certain empirical dualism between these two types of theorems: As soon as a theorem of type $(H)$ is discovered, its wording and proof can usually be altered so as to supply a theorem of type $(L)$, and conversely. But as far as the literature consulted goes, this principle of transfer appears only ad hoc, since its applicability is not assured $a$ priori.

The following consideration will dispose of this anomaly, by exhibiting a formal fact which underlies the principle of tranfer, $(H) \rightarrow(L)$ or $(L) \rightarrow(H)$.

4. It will turn out that what actually matters in this regard is neither (2) nor (3) but a connection between the differential equations (1) and.

$$
\ddot{x}+g(s) x=0,
$$

where the dots, in contrast to the primes of (1), refer to differentiations with respect to the independent variable $s=s(t)$ defined by

$$
d s=f(t) d t
$$

${ }^{2}$ Concerning the brackets see the preceding footnote.

scf. G. Sansone, Equazioni differenziali nel campo reale, vol. 2 (2nd ed., 1949), chap. VII, and F. G. Tricomi, Equazioni differenziali (2nd ed., 1953), chap. III-IV. These expositions, and the papers mentioned in the first footnote above, contain further references to the literature. 
and the coefficient functions of (1) and (6) are reciprocals by virtue of (7) or

that is,

$$
d t=d s / g(s)
$$

$$
f=1 / g \text { or } g=1 / f \text {. }
$$

Note that, because of (4) and (7), the correspondence $s=s(t)$ between the given $t$-range and the $s$-range is a one-to-one correspondence, which is continuously differentiable along with its inverse, $t=t(s)$, and that, by virtue of (4), (7) and (5),

$$
t \rightarrow \infty \text { if and only if } s \rightarrow \infty \text {. }
$$

It is clear from (9) that (6) is of low frequency or of high frequency according as (1) is of high frequency or of low frequency. But the connection between (1) and (6), being of a formal nature, will be such as to apply also in the intermediary cases, cases in which neither (2) nor (3) is satisfied.

5. The proof depends, as in [1], on an appropriate application of Riccati's equation (an application which, however, cannot be the same as in [1]; cf. Section 6 below).

First, if $I$ is a $t$-interval on which a solution $x(t)$ of (1) does not vanish, then it is possible to form the logarithmic derivative

$$
m(t)=x^{\prime}(t) / x(t)
$$

at every point $t$ of $I$, and $m=m(t)$ will be a solution of Riccati's differential equation

$$
m^{\prime}+m^{2}+f(t)=0 .
$$

In fact, if (1) is divided by $x$, then (12) follows from (11).

Next, suppose that not only $x(t)$ but also $x^{\prime}(t)$ is distinct from 0 at every point $t$ of $I$. Then (11) shows that it is possible to form on $I$ the function

$$
n(t)=-1 / m(t),
$$

and it is seen from (13) that (12) is equivalent to

$$
n^{\prime}+1+f(t) n^{2}=0 .
$$

Finally, if (14) is divided by $f(t)$ (which, in view of (4), is allowed), then the definitions, (9) and (7), of $s$ and $g$ show that (14), where $n^{\prime}=d n / d t$, can be written in the form

$$
\dot{n}+n^{2}+g(s)=0,
$$

where $\dot{n}=d n / d s$.

This proves that, as a matter of formal coincidence, (1) is equivalent to (6) by virtue of (9) and (7). In fact, (15) belongs to (6) in the same way (that is, as the Riccati resolvent of the linear differential equation) as (12) belongs to (1).

6. Actually, the proof is not yet complete. For, on the one hand, Section 5 confines $t$ to a $t$-range $I$ on which neither $x(t)$ nor $x^{\prime}(t)$ has a zero and, on the other hand, the problems of the asymptotic behavior concern themselves with the $t$-range $I$ which is a half-line $t_{0} \leqq t<\infty$ on which $x(t)$ (hence $x^{\prime}(t)$ as well) has an infinity of zeros, since (1) is oscillatory. In fact, the oscillatory character of (1) is implied by (4) and (5), and (5) cannot be omitted, since otherwise (10) becomes false. This difficulty can however readily be disposed of, as follows: 
Instead of a single real-valued solution $x(t)$ of (1), choose for (1) two solutions, $x=x(t)$ and $x=y(t)$, which are real-valued and linearly independent. In view of the superposition principle, the (complex-valued) function

$$
z(t)=x(t)+i y(t)
$$

is a solution of the (real) differential equation (1). But

$$
z(t) \neq 0 \text { and } z^{\prime}(t) \neq 0
$$

now hold on $I$ when $I$ is the entire half-line under consideration $\left(t_{0} \leqq t<\infty\right)$. In fact, since $x=x(t)$ and $x=y(t)$ are linearly independent solutions of (1), their Wronskian $x y^{\prime}-y x^{\prime}$ is a non-vanishing constant; hence neither $x$ and $y$ nor $x^{\prime}$ and $y^{\prime}$ can vanish at the same $t$, and so it is clear from (16) that both inequalities (17) are satisfied at every $t$.

Owing to the first of the inequalities (17), the logarithmic derivative

$$
m(t)=z^{\prime}(t) / z(t)
$$

exists on the entire half-line $I$. On the other hand, the second of the inequalities (17) assures that (13) defines the function $n(t)$ on the entire half-line $I$ if $m(t)$ is defined by (18).

Finally, since $x=z(t)$ is a solution of (1), Riccati's deduction of (12) from (11) and the case $x=x(t)$ of (1) remains unaltered if (11) and (1) are replaced by (17) and the case $x=z(t)$ of (1) respectively. Since the transition from (12) to (15) had nothing to do with the reality of $m(t)$, the proof is now complete.

\section{ON THE ROTATION OF AN INCOMPRESSIBLE CONTINUOUS MEDIUM IN PLANE MOTION*}

\section{BY WALTER NOLL (Carnegie Institute of Technology)}

We consider a plane motion of an incompressible medium with homogeneous density $\rho=$ const. We assume that the plane region occupied by the medium is of the following type: at each instant it is bounded by a finite number of smooth curves. If it is finite all but one of the closed boundary curves move rigidly. If it extends to infinity all finite closed boundary curves move rigidly. We label this motion by I. Now we consider another motion, labeled by II, which is obtained from I by superposing a uniform rotation with angular velocity $\omega=$ const. about a fixed axis perpendicular to the plane of the motion.

It will be shown that motion II is dynamically possible whenever I is, and that the stresses in II differ from those in I only by a scalar stress given explicitly in (10) below.

G. I. Taylor [1] proved this theorem for the special case of the irrotational motion of an inviscid fluid caused by rigid cylindrical bodies moving in an infinite container. It has been shown recently that Taylor's result continues to hold for linear viscous fluids [2] and for a special type of non-linear viscous fluids [4]. The main purpose of this paper is to point out that Taylor's theorem remains valid for any material whatsoever (it may even be an anisotropic solid). The essential assumptions are that the medium be incompressible and that the motion be plane.

${ }^{*}$ Received August 17, 1956. Written under Contract Nonr 228(09), Office of Naval Research. 